Article

\title{
In-silico study of the immune systems associated genes in case of type-2 diabetes with Insulin action and resistance and in obesity
}

\author{
Basmah Medhat Eldakhakhny ${ }^{1}$, Hadeel Al Sadoun ${ }^{2}$, Hani Choudhry, ${ }^{3, *}$, and Mohammad \\ Mobashir ${ }^{4, *}$ \\ 1 Department of Clinical Biochemistry, Faculty of Medicine, King Abdulaziz University , Jeddah , Saudi \\ Arabia; Email: beldakhakhny@kau.edu.sa \\ 2 Department of Medical Laboratory Technology, Faculty of Applied Medical Sciences, King Abdulaziz \\ University, Saudi Arabia; Email: hsadounkau.ed.sa@kau.edu.sa \\ 3 Department of Biochemistry, Cancer Metabolism and Epigenetic Unit, Faculty of Science, Cancer and \\ Mutagenesis Unit, King Fahd Medical Research Center, King Abdulaziz University, Jeddah 21589, Saudi \\ Arabia; Email: hchoudhry@kau.edu.sa \\ 4 Department of Microbiology, Tumor and Cell Biology (MTC) Karolinska Institute, Novels väg 16, 17165 \\ Solna, Stockholm, Sweden Tel: +46 70872 3675; Email:m.mobashir@cdslifesciences.com \\ * Correspondence: hchoudhry@kau.edu.sa and m.mobashir@cdslifesciences.com; Tel: +46 708723675
}

\begin{abstract}
Type-2 diabetes and obesity are among the leading human diseases and highly complex in terms of diagnostic and therapeutic approaches and are among the most frequent and highly complex and heterogeneous in nature. Based on epidemiological evidence, it is known that the patients suffering from obesity are considered to be significantly at higher risk for type-2 diabetes. There are a number of evidences which support the hypothesis that these diseases interlinked and obesity may aggravate the risk(s) of type-2 diabetes. Multi-level unwanted alterations such as (epi) genetic alterations, changes at the transcriptional level, and altered signaling pathways (receptor, cytoplasmic, and nuclear level) are the major source which promotes a number of complex diseases and such heterogeneous level of complexities are considered as the major barrier in the development of therapeutic. With so many known challenges, it is critical to understand the relationships and the common shared causes between type- 2 diabetes and obesity which is difficult to unravel and understand. For this purpose, we have selected publicly available datasets of gene expression for obesity and type- 2 diabetes and unraveled the genes and the pathways associated with immune system and have also focused on $\mathrm{T}$ cell signaling pathway and its components. We have applied simplified computational approach to understand differential gene expression and patterns and the enriched pathways for obesity and type-2 diabetes. Furthermore, we have also analyzed the genes by using network-level understanding. In the analysis, we observe that there are less number of genes which are commonly differentially expressed while comparatively higher number of pathways are shared between them. There are only four pathways which are associated with immune system in case of obesity and ten immune-associated pathways in case of type-2 diabetes and among them only two pathways are commonly altered.
\end{abstract}

Keywords: Type- 2 diabetes; obesity, insulin action and resistance; immune system; enriched pathways, differentially expressed genes; TCR network; network-level understanding.

\section{Introduction}

Type 2 diabetes (T2D) is a global epidemic, strongly correlated which is more common among the obese[1,2]. Adipose tissue inflammation associated with obesity is known as a major cause for the decreased insulin sensitivity in case of T2D $[1,3,4]$. There are a number of works where the crosstalk between the immune system and metabolism have been presented and a number of evidences which 
support the hypothesis that these diseases interlinked and obesity may aggravate the risk(s) of T2D[58]. In such cases, it is critical to understand multi-level unwanted alterations such as (epi-)genetic alterations, changes at the transcriptional level, and altered signaling pathways (receptor, cytoplasmic, and nuclear level). These are the major source which promotes a number of complex diseases and such heterogeneous level of complexities are considered as the major barrier in the development of therapeutic approach(es). With such diverse challenges, it is crucial to understand and unravel the relationships and the common shared causes between type-2 diabetes and obesity[913].

So far, there are a number of previous study where a number of factors have been investigated to understand the biological mechanism of obesity and T2D and the relationship between each other[1,2,14-16]. T2D is mainly characterized by a progressive status of chronic and inflammation of low grade and inflammatory responses are triggered by many factors. The well-known factors contributing to T2D are age, metabolic syndrome, systematic low-grade inflammation, insulin resistance, islet cell auto-antibodies, beta cell dysfunction, and C-peptide. Some of the factors which promotes inflammation are altered function of specific T lymphocyte cells, B lymphocytes, Th1, and Th17[17-21]. As mentioned above that T2D is also linked with obesity and these diseases are also the case of metabolic disorder and there exist strong evidences of immune-metabolic connection[10,22,23].

For this purpose, we have selected publicly available datasets of gene expression for obesity and T2D and unraveled the genes and the pathways associated with immune system and have also focused on T cell signaling pathway and its components. We have applied simplified computational approach to understand differential gene expression and patterns and the enriched pathways for obesity and T2D. Furthermore, we have also analyzed the genes by using network-level understanding. In the analysis, we observe that there are less number of genes which are commonly differentially expressed while comparatively higher number of pathways are shared between them. There are only four pathways which are associated with immune system in case of obesity and ten immune-associated pathways in case of T2D and among them only two pathways are commonly altered. Here, we have studied the differentially expressed genes (DEGs), enriched pathways, and the connection between immune signaling pathways via their components between obesity and T2D and with the major focus on the roles TCR signaling components.

\section{Results}

As mentioned above that we have compared immune signaling genes by using gene expression profiling of T2D and obese for which the datasets have been collected from publicly available data. Here, the results have been presented from work from generalized analysis (i.e., DEGs, enriched pathways) to precise analysis i.e., TCR signaling. 


\subsection{Gene expression profiling for type-2 diabetes and obesity}

For diabetes and kidney cancer collected data, we have performed comparative analysis in terms of altered gene expression pattern and their respective functions. Here, we have presented the DEGs for both the cases T2D and obesity (Figure 1a) and we observe that there are only 12 genes which are differentially expressed in both the cases while T2D-specific genes are 456 and 580 genes are obesityspecific genes while in terms of the altered functions they share 14 pathways and the T2D and obesity specific pathways are 17 and 18 (Figure $1 \mathrm{~b}$ and Table 1), respectively. Based on this it appears that there are only few genes which differentially expressed in both the cases while in terms of biological functions, a large number of pathways are affected which means that T2D and obesity share more biological functions in terms of alterations and their source of alterations i.e., DEGs are not shared at large scale. The green color nodes are the DEGs which either belong to T2D or obesity case. For this reason, we have mapped out the network for common DEGs and highlighted common DEGs in red color (Figure 1c). Among these common DEGs, FN1, SFRP1, and SNX5 appears to be the major source of alteration. FN1 appears to be associated with a large number of DEGs leading to the conclusion that either FN1 is overexpressed by those genes or it promotes the over expression of the directly associated genes. For understanding the alteration in biological function, a heatmap has been displayed in Figure 1d for those pathways which are commonly enriched. For this plot, the p-values in both the conditions have been used. Cell adhesion molecules, PI3K-AKT, and MAPK pathways appear highly enriched in both the conditions, regulation of actin cytoskeleton, cAMP, phospholipase D, and Focal adhesion are exclusively and highly enriched in case of T2D, and hematopoetic cell lineage and phagosome are potentially enriched mainly in case of obesity. There are additional pathways which are enriched in both the cases but have comparatively higher p-values and these pathways are Rap1, tight junction, leukocyte transendothelial migration, ECM-receptor interaction, and Ras signaling pathways. The complete details about the fold changes of the genes have been presented in supplementary data S1 and S2.

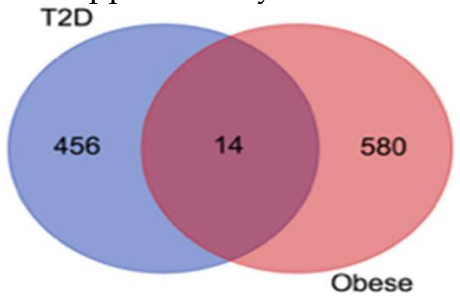

(a)

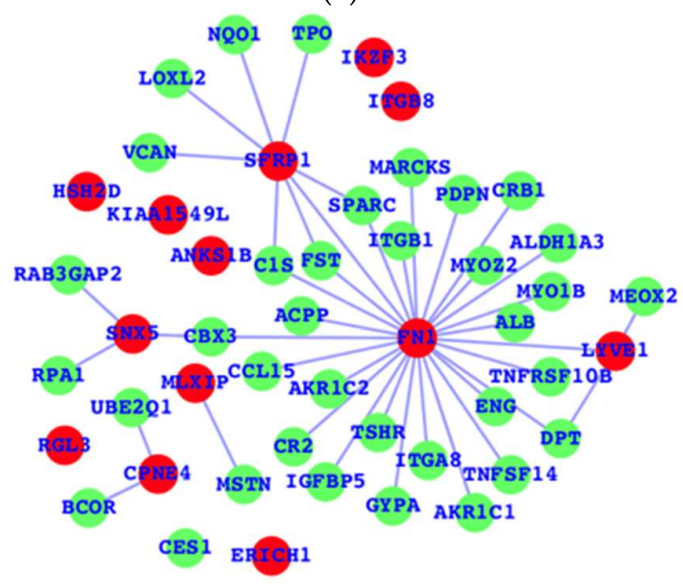

(c)

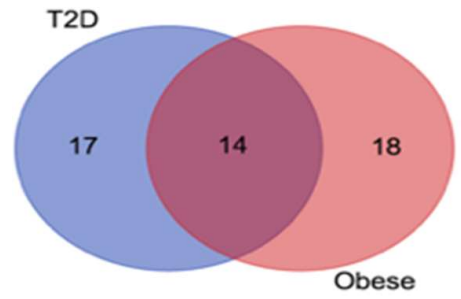

(b)

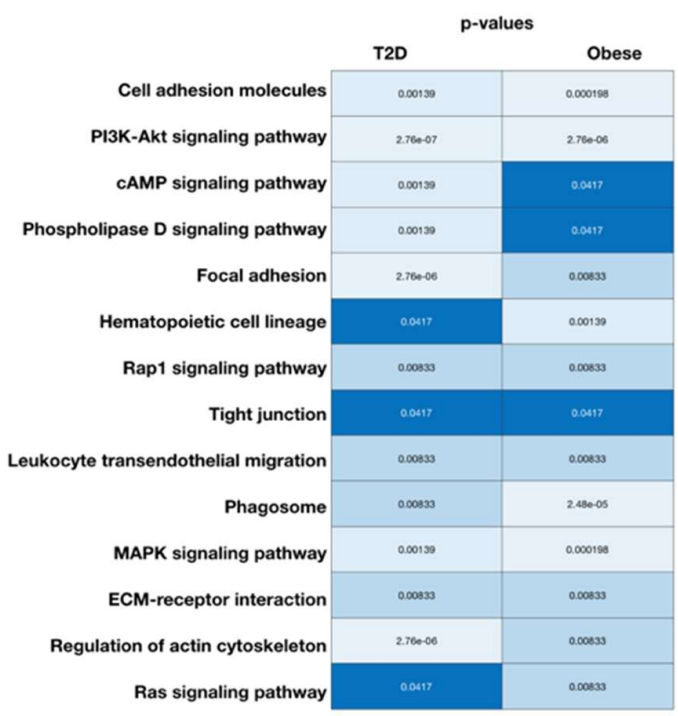

(d) 
Figure 1. Gene expression profiling. (a) Venn diagram for differentially expressed genes. (b) Venn diagram for enriched pathways. (c) Network representing the DEGs common between T2D and obesity. (d) Commonly enriched pathways.

Table 1. Pathways common between T2D and obesity and specific to T2D and obesity (venn diagram outcome).

\begin{tabular}{|c|c|c|}
\hline Name & & Pathways \\
\hline $\begin{array}{c}\mathrm{T} 2 \mathrm{D} \\
\text { and } \\
\text { Obese }\end{array}$ & 14 & $\begin{array}{l}\text { Cell adhesion molecules (CAMs); PI3K-Akt signaling pathway; cAMP signaling } \\
\text { pathway; Phospholipase D signaling pathway; Focal adhesion; Hematopoietic cell } \\
\text { lineage; Rap1 signaling pathway; Tight junction; Leukocyte transendothelial } \\
\text { migration; Phagosome; MAPK signaling pathway; ECM-receptor interaction; } \\
\text { Regulation of actin cytoskeleton; Ras signaling pathway }\end{array}$ \\
\hline T2D & 17 & $\begin{array}{l}\text { Neurotrophin signaling pathway; Insulin signaling pathway; Vascular smooth } \\
\text { muscle contraction; Sphingolipid signaling pathway; cGMP-PKG signaling } \\
\text { pathway; mTOR signaling pathway; T cell receptor signaling pathway; Natural } \\
\text { killer cell mediated cytotoxicity; Jak-STAT signaling pathway; Progesterone- } \\
\text { mediated oocyte maturation; B cell receptor signaling pathway; Oxytocin } \\
\text { signaling pathway; Platelet activation; Thyroid hormone signaling pathway; Wnt } \\
\text { signaling pathway; ErbB signaling pathway; Ubiquitin mediated proteolysis }\end{array}$ \\
\hline Obese & 18 & $\begin{array}{l}\text { Fatty acid metabolism; Cell cycle; Tyrosine metabolism; Thyroid hormone } \\
\text { synthesis; Starch and sucrose metabolism; Purine metabolism; Cytokine-cytokine } \\
\text { receptor interaction; Metabolism of xenobiotics by cytochrome P450; Retinol } \\
\text { metabolism; Drug metabolism - cytochrome P450; Neuroactive ligand-receptor } \\
\text { interaction; Tryptophan metabolism; p53 signaling pathway; Complement and } \\
\text { coagulation cascades; Antigen processing and presentation; Arachidonic acid } \\
\text { metabolism; Axon guidance; Retrograde endocannabinoid signaling }\end{array}$ \\
\hline
\end{tabular}

\subsection{Genes associated with immune system}

After analyzing the overall DEGs and the all the enriched pathways, we have performed immune system specific analysis for the genes and the pathways. Here, we observe that there are 10 pathways associated with immune system in case of T2D while there are four pathways in case of obese which are associated with immune system (Figure 2). Hematopoetic cell lineage and leukocyte transendothelial migration pathways are commonly altered in both the cases T2D and obese and these two pathways are known to play roles blood cell development and immune survilliance and inflammation leading to the conclusion that in both the cases T2D and obesity, the blood cell development, immune survilliance, and the inflammation process are affected. Obesity-specific immune signaling pathways are oxytocin and antigen processing and presentation (APP). Oxytocin signaling pathway is known to exert a wide range of effects which could be central or peripheral effects and this pathway itself is known to crosstalk with a number of critical pathways such as RhoA, GPCR, MAPK, ANP-cGMP, and NO-cGMP. APP is one of the most important part of entire immune system and is the process by which protein antigen is ingested by an antigen-presenting cell (APC), partially digested into peptide fragments and then displayed on the surface of the APC associated with an antigen-presenting molecule (MHC class I or MHC class II), for recognition by specific lymphocytes (T cells). While there are eight immune system specific pathways in case of T2D which are NK cell-mediated cytotoxicty, insulin signaling, TCR signaling, ubiquitin-mediated proteolysis, platelet activation BCR signaling, neurotrophin, and oxytocin signaling pathways. Almost all these pathways are major part of immune system. Based on this, we could conclude that in case of T2D, there is more alterations in the gene expression pattern of those genes which are known to control major part of immune system while in case of obesity there is less effect than T2D but these few pathways are also known to be associated with controlling critical roles of immune system. 


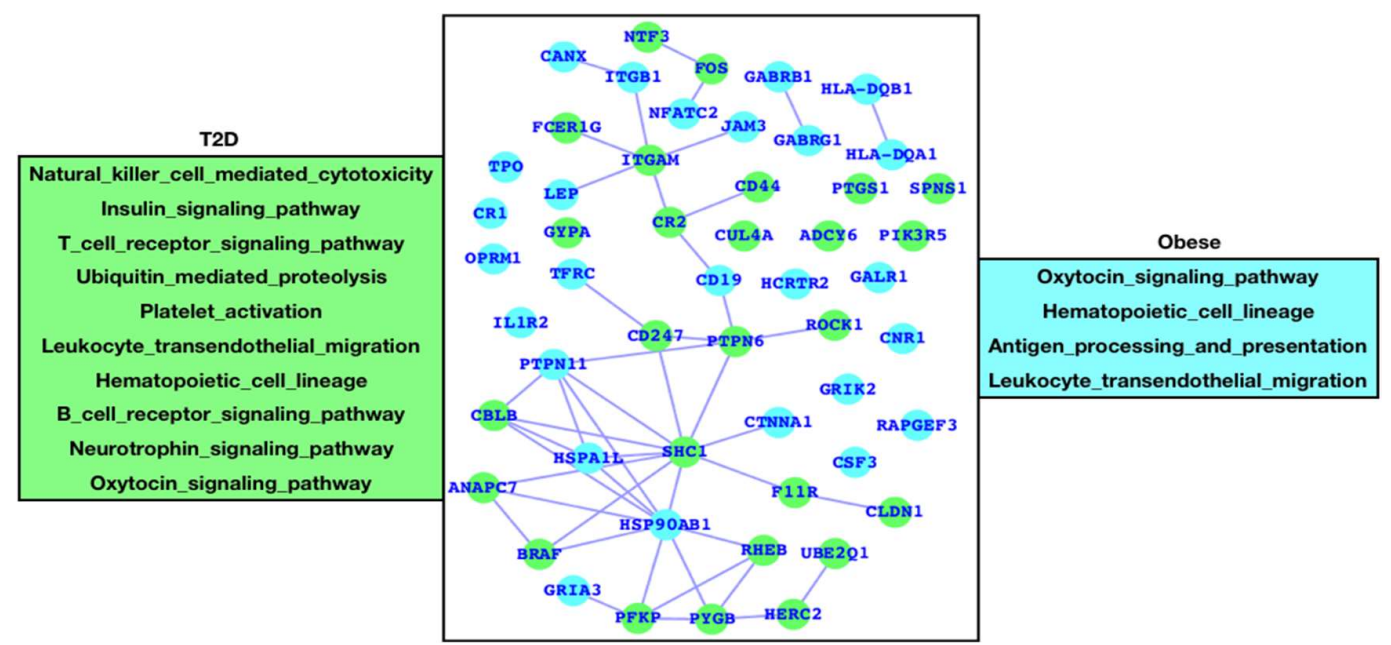

Figure 2. Genes associated with immune systems. Green color genes belong to the immune system when the patient is suffering from T2D and cyan color genes belong to the immune system in case of obese.

\subsection{DEGs of T2D and TCR signaling network}

As mentioned above that there are a number of pathways which are affected as a result of T2D and obesity while in comparison to T2D, because of obesity there are only four pathways which are mainly affected and TCR signaling pathway is not affected here. TCR signaling pathway is affected in case of T2D. Therefore, we have now mapped out the individual genes which are associated with TCR signaling pathway and presented the networks for all the five genes (SPNS1, PTPN6, CD247, FOS, and PIK3R5) which are the component of TCR signaling pathway and their association with the other TCR genes (Figure 3a-3e). In addition, we have also presented the TCR network (Figure 3f).

SPNS1 gene is the direct component of TCR, NK cell mediated cytotoxicity, and FC-epsilon RI signaling pathways, PTPN6 belongs to TCR, BCR, NK cell-mediated cytotoxicity, JAK-STAT, and adherens junction, CD247 belongs to TCR and NK cell-mediated cytotoxicity, and FOS belongs to TCR, BCR, TLR, MAPK, TNF, cAMP, estrogen, oxytocin, prolactin, circadian entrainment, and osteoclast differentiation. PIK3R5 alone belongs to a large number of critical pathways such as TCR, BCR, NK cell-mediated cytotoxicity, JAK-STAT, ErbB, chemokine, phosphatidylinositol, mTOR, apoptosis, VEGF, focal adhesion, regulation action cytoskeleton, etc., and the complete details of the list of pathways for all these five genes have been presented in Table 2 . 


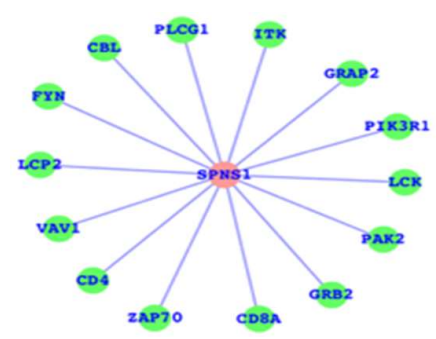

(a)

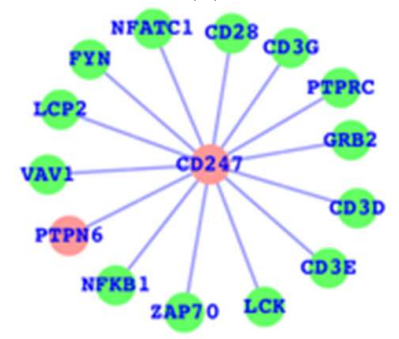

(c)

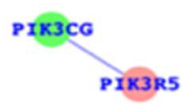

(e)

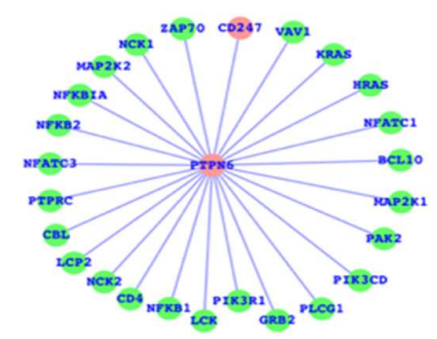

(b)

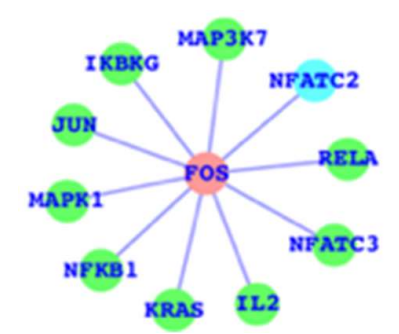

(d)

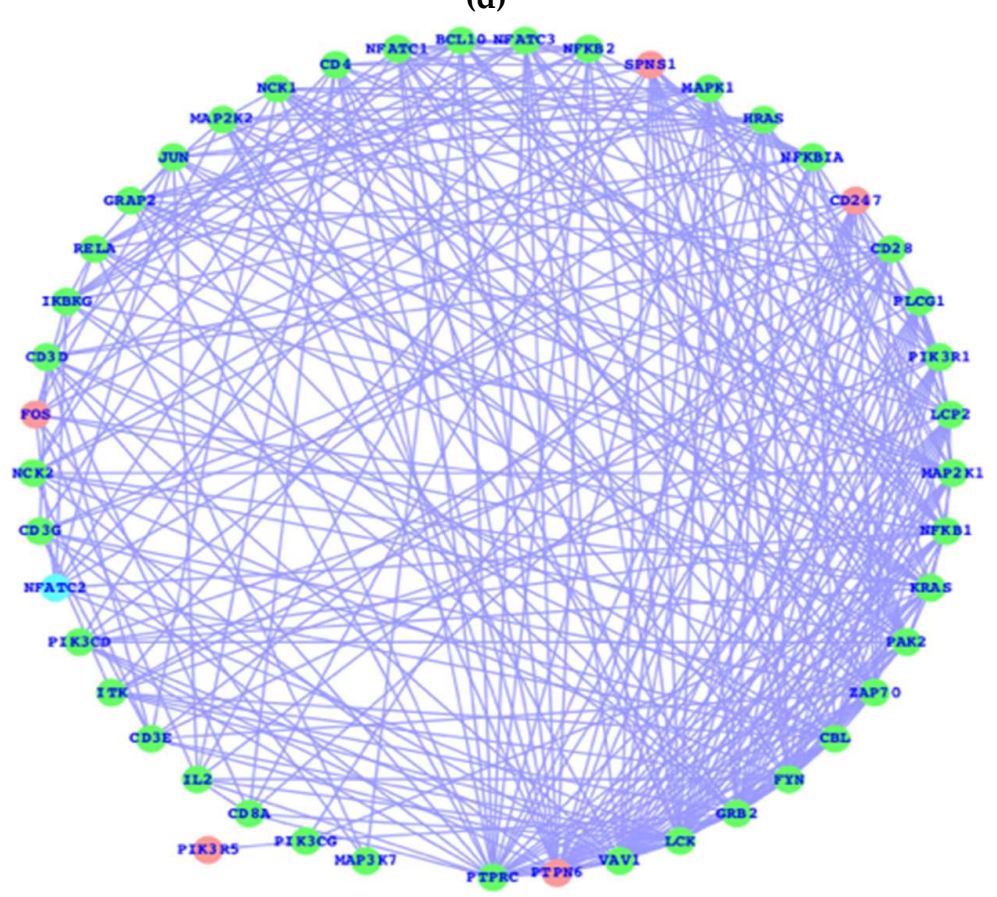

(f)

Figure 3. DEGs of T2D and TCR signaling network. (a) SPNS1 network, (b) PTPN6 network, (c) CD247 network, (d) FOS network, (e) PIK3R5 network, and (f) Overall association of these T2D DEGs with the TCR genes and also the intra-associations.

Table 2. Selected gene specific pathways.

\begin{tabular}{cl}
\hline Gene & \multicolumn{1}{c}{ Pathways } \\
\hline \multirow{3}{*}{ SPNS1 } & T cell receptor signaling pathway \\
& Fc epsilon RI signaling pathway \\
& Natural killer cell mediated cytotoxicity \\
PTPN6 & Adherens junction \\
& Jak-STAT signaling pathway \\
\hline
\end{tabular}


Natural killer cell mediated cytotoxicity

$\mathrm{T}$ cell receptor signaling pathway

B cell receptor signaling pathway

CD247

Natural killer cell mediated cytotoxicity

$T$ cell receptor signaling pathway

MAPK signaling pathway

Toll-like receptor signaling pathway

$\mathrm{T}$ cell receptor signaling pathway

B cell receptor signaling pathway

TNF signaling pathway

FOS cAMP signaling pathway

Osteoclast differentiation

Circadian entrainment

Estrogen signaling pathway

Prolactin signaling pathway

Oxytocin signaling pathway

ErbB signaling pathway

Chemokine signaling pathway

Phosphatidylinositol signaling system

mTOR signaling pathway

Apoptosis

VEGF signaling pathway

Focal adhesion

Toll-like receptor signaling pathway

Jak-STAT signaling pathway

Natural killer cell mediated cytotoxicity

$\mathrm{T}$ cell receptor signaling pathway

B cell receptor signaling pathway

Fc epsilon RI signaling pathway

Fc gamma R-mediated phagocytosis

Leukocyte transendothelial migration

Neurotrophin signaling pathway

Regulation of actin cytoskeleton

Insulin signaling pathway

Progesterone-mediated oocyte

maturation

Type II diabetes mellitus

Aldosterone-regulated sodium

reabsorption

Bacterial invasion of epithelial cells

\section{Discussion}


Diabetes mellitus (DM) and obesity are considered as metabolic disorder related diseases and $\mathrm{DM}$ is characterized by hyperglycemia resulting from defects in insulin secretion, insulin action, or both[1,24,25]. The diabetic chronic hyperglycemia is associated with long-term damage, dysfunction, and failure of various organs such as kidneys, eyes, nerves, blood vessels, and heart[1,17,25-29]. T2D is most often found to be frequent in case of the obese and is characterized by abnormal insulin secretion and/or a decreased sensitivity for insulin which is also known as insulin resistance and results in increased blood glucose levels and T2D is known to be strongly associated with obesity. There are a number of biological processes and mechanisms which are considered as the source of association between these conditions via mediating inflammation in adipose tissue and systemic insulin resistance such as oxidative stress, endoplasmic reticulum stress, hypoxia, amyloid and lipid deposition, lipotoxicity and glucotoxicity.

With such level of complexities, there exist a number of challenges for understanding the mechanism of so we have thoroughly analyzed the DEGs, enriched pathways with major focus on immune signaling pathways and the networks of DEGs belonging to the immune system. Among the top enriched pathways are: cell adhesion molecules, PI3K-AKT, MAPK, regulation of actin cytoskeleton, cAMP, phospholipase D, Focal adhesion, hematopoetic cell lineage, phagosome, Rap1, tight junction, leukocyte transendothelial migration, ECM-receptor interaction, and Ras signaling pathways.

For immune systems - related analysis, we observe that there are 10 pathways associated with immune system in case of T2D while there are four pathways in case of obese which are associated with immune system. Hematopoetic cell lineage and leukocyte transendothelial migration pathways are commonly altered in both the cases T2D and obese and are known to play roles blood cell development and immune survilliance and inflammation. Oxytocin and APP are obesity-specific immune signaling pathways and NK cell-mediated cytotoxicty, insulin signaling, TCR signaling, ubiquitin-mediated proteolysis, platelet activation BCR signaling, neurotrophin, and oxytocin signaling pathways are T2D immune system specific pathways. Almost all these pathways (obese and T2D immune signaling pathways) are major part of immune system known to control major part of immune system. Furthermore, it is also known that altered TCR signaling is associated with T2D. Therefore, we have now mapped out the individual genes which are associated with TCR signaling pathway and presented the networks for all the five genes (SPNS1, PTPN6, CD247, FOS, and PIK3R5) which are the component of TCR signaling pathway and their association with the other TCR genes (Figure 3). These five genes SPNS1, PTPN6, CD247, FOS, and PIK3R5 are the potential components of TCR and also a number of additional immune system associated pathways. Among those pathways are NK cell mediated cytotoxicity, FC-epsilon RI signaling pathways, BCR, JAK-STAT, adherens junction, TLR, MAPK, TNF, cAMP, estrogen, oxytocin, prolactin, circadian entrainment, and osteoclast differentiation. PIK3R5 alone belongs to a large number of critical pathways such as TCR, BCR, NK cell-mediated cytotoxicity, JAK-STAT, ErbB, chemokine, phosphatidylinositol, mTOR, apoptosis, VEGF, focal adhesion, regulation action cytoskeleton, etc[30].

As we can see in the results that PIK3R5 and FOS which are the components of TCR signaling pathway and also control a large set of biological functions most of them part of immune system and CD247, PTPN6, and SPNS1 comparatively controls less number of pathways but in terms of connectivity PTPN6 has the highest connectivity then SPNS1, CD247, FOS, and PIK3R5. It means that PIK3R5 simultaneously controls large number of biological functions but does not impact more genes or in other words the higher connectivity of the gene will have more chance to affect the expression patterns of the gene or vice versa. In terms of future perspective, this study will be helpful for therapeutic approach also for drug targeting.

\section{Materials and Methods}

For obese, GSE12050[31] dataset used where there are 36 samples out of which nine lean needle biopsy have been compared with 9 obese biopsy samples. For sample details, it has been mentioned that abdominal subcutaneous fat specimens have been collected by using needle biopsy approach 
from nine obese patient samples and 10 lean samples. Then nine obese patient samples have been collected from abdominal subcutaneous fat biopsies and nine lean samples from abdominal fat biopsies[31]. For T2D, GSE121 (GPL101 platform)[32] has been used for type-2 diabetes gene expression profiling which contains 5 (insulin resistant (IR)) and 5 samples (Insulin sensitive (IS)) are with type II diabetes while 11 are control samples. The samples were non-diabetic Pima Indians or Tohono O'Odham Indians who were classified as IS or IR[32].

These gene expression datasets were collected from GEO have been processed for expression value calculation, fold change, and p-value calculation by the in-built code at GEO i.e., GEO2R [33,34]. The list of DEGs have been processed for pathway enrichment analysis and understanding[35,36]. KEGG[30] database have been used for calculating enriched pathways and further, own developed code have been used for pathway and network analysis. To retrieve DEGs association as a network, FunCoup2.0 network database[37] has been utilized throughout the work and the network has been visualized with the help of cytoscape[38]. For most of our coding and calculations MATLAB has been used.

Now, we briefly summarize all the steps used are raw file processing, intensity calculation and normalization. Since, the high-throughput data mainly microarray-based gene expression values are calculated as intensity which ranges from lowest values to extremely high so it is needed to have optimized values or scaled down values so we normally convert them to $\log 2$ values. For normalization[39-41], GCRMA[42-46], RMA, and EB are the most commonly used approaches. Here, we have used EB for raw intensity normalization. After normalization, we proceed for our goal which is to understand the gene expression patterns[35,47] and its inferred functions[35,36]. For differential gene expression prediction and statistical analysis, MATLAB functions (e.g., mattest) has been used and volcano plot. From mattest, we get the p-values and then we go for p-values corrections and assign the p-value threshold and finally volcano plot has been used for fold change calculation and the selection of genes with $p$-values less than 0.05 and fold changes within this range $>=+2.0$ and $<=$ -2.0 and this range of fold change as well as the p-value cutoff are commonly used while in our study we have selected the top ranked genes to have strict condition of selection based on fold change so the fold changes are either extremely high or extremely low than the cutoff fold change. For pathway analysis, we used KEGG[30] database and have our own code designed to pathway and network analysis[48-50]. We have described about the fold change and by default for considering genes as DEGs, we have applied the threshold for FC $>=+2.0$ (up regulated genes) and FC $<=-2.0$ (down regulated genes) and these DEGs have been considered for pathways enrichment analysis. But for heatmap and network presentations the top 100 DEGs (50 up and 50 down) genes have been presented[51,52].

\section{Conclusions}

Here, we observe that there are less number of genes which are commonly differentially expressed while comparatively higher number of pathways are shared between them. There are only four pathways which are associated with immune system in case of obesity and ten immuneassociated pathways in case of T2D and among them only two pathways are commonly altered. Here, we have studied the differentially expressed genes (DEGs), enriched pathways, and the connection between immune signaling pathways via their components between obesity and T2D and with the major focus on the roles TCR signaling components. We conclude that in case of T2D, there is more alterations in the gene expression pattern of those genes which are known to control major part of immune system while in case of obesity there is less effect than T2D but these few pathways are also known to be associated with controlling critical roles of immune system.

Author Contributions: “Conceptualization, B.M.E., H.A.S., H.C., and M.M.; methodology, B.M.E., H.A.S., H.C., and M.M.; software, M.M.; validation, B.M.E., H.A.S., H.C., and Z.Z.; formal analysis, B.M.E., H.A.S., H.C, and 
M.M.; investigation, B.M.E. and M.M.; resources, B.M.E. and M.M.; data curation, B.M.E. and M.M.; writingoriginal draft preparation, B.M.E., H.A.S., H.C., and M.M.; writing-review and editing, B.M.E., H.A.S., H.C., and M.M.; visualization, B.M.E., H.A.S., H.C., and M.M.; supervision, H.C. and M.M.; project administration, B.M.E. and H.C.; funding acquisition, B.M.E. All authors have read and agreed to the published version of the manuscript."

Funding: "This research was funded by The Deanship of Scientific Research (DSR) at King Abdulaziz University, Jeddah, grant number RG-5-140-39" and "The APC was funded by RG-5-140-39".

Acknowledgments: The Deanship of Scientific Research (DSR) at King Abdulaziz University, Jeddah, funded this project, under grant number RG-5-140-39. We are also thankful to King Abdulaziz University for offering us the environment to carry out the entire research work and all other supports.

Conflicts of Interest: Declare conflicts of interest or state "The authors declare no conflict of interest." "The funders had no role in the design of the study; in the collection, analyses, or interpretation of data; in the writing of the manuscript, or in the decision to publish the results".

\author{
Abbreviations \\ DEGs Differentially expressed genes \\ TCR T cell receptor \\ ISPs Immune signaling pathways \\ T2D Type-2 diabetes \\ APP Antigen processing and presentation \\ APC Antigen-presenting cell
}

\title{
Appendix A
}

The appendix is an optional section that can contain details and data supplemental to the main text. For example, explanations of experimental details that would disrupt the flow of the main text, but nonetheless remain crucial to understanding and reproducing the research shown; figures of replicates for experiments of which representative data is shown in the main text can be added here if brief, or as Supplementary data. Mathematical proofs of results not central to the paper can be added as an appendix.

\section{Appendix B}

All appendix sections must be cited in the main text. In the appendixes, Figures, Tables, etc. should be labeled starting with 'A', e.g., Figure A1, Figure A2, etc.

\section{References}

1. Sacerdote, C.; Ricceri, F. Accepted Manuscript. Diabetes Research and Clinical Practice 2018, 1-30.

2. Arbeeny, C. M. Metabolic Diseases: Biological Mechanisms; John Wiley \& Sons, Inc.: Hoboken, NJ, USA, 2007.

3. Nikolajczyk, B. S.; Jagannathan-Bogdan, M.; Shin, H.; Gyurko, R. State of the union between metabolism and the immune system in type 2 diabetes. Genes and Immunity 2019, 1-12.

4. Shu, C. J.; Benoist, C.; Mathis, D. Seminars in Immunology. Seminars in Immunology 2012, 24, 436-442.

5. Kaarbø, M.; Klokk, T. I.; Saatcioglu, F. Androgen signaling and its interactions with other signaling pathways in prostate cancer. Bioessays 2007, 29, 1227-1238.

6. Ma, Y.; Galluzzi, L.; Zitvogel, L.; Kroemer, G. Autophagy and Cellular Immune Responses. Immunity 2013, $39,211-227$.

7. Jee, S. H.; Kim, H. J.; Lee, J. Obesity, insulin resistance and cancer risk. Yonsei Med. J. 2005, 46, 449-455.

8. Szablewski, L. From obesity through immunity to type 2 diabetes mellitus. International Journal of Diabetes in Developing Countries 2016, 1-12.

9. Jaikaran, E. T.; Clark, A. Islet amyloid and type 2 diabetes: from molecular misfolding to islet pathophysiology. Biochim. Biophys. Acta 2001, 1537, 179-203. 
10. Richard, C.; Wadowski, M.; Goruk, S.; Cameron, L.; Sharma, A. M.; Field, C. J. Individuals with obesity and type 2 diabetes have additional immune dysfunction compared with obese individuals who are metabolically healthy. BMJ Open Diab Res Care 2017, 5, e000379.

11. Zhou, T.; Hu, Z.; Yang, S.; Sun, L.; Yu, Z.; Wang, G. Review ArticleRole of Adaptive and Innate Immunity in Type 2 Diabetes Mellitus. Journal of Diabetes Research 2018, 1-9.

12. Murea, M.; Ma, L.; Freedman, B. I. Genetic and environmental factors associated with type 2 diabetes and diabetic vascular complications. Rev Diabet Stud 2012, 9, 6-22.

13. Pickup, J. C. Inflammation and activated innate immunity in the pathogenesis of type 2 diabetes. Diabetes Care 2004, 27, 813-823.

14. Klil-Drori, A. J.; Azoulay, L.; Pollak, M. N. Cancer, obesity, diabetes, and antidiabetic drugs: is the fog clearing? Nat Rev Clin Oncol 2016, 1-15.

15. Draznin, B. Molecular Mechanisms of Insulin Resistance: Serine Phosphorylation of Insulin Receptor Substrate-1 and Increased Expression of p85 : The Two Sides of a Coin. Diabetes 2006, 55, 2392-2397.

16. Pathogenesis of. 2010, 1-54.

17. de Candia, P.; Prattichizzo, F.; Garavelli, S.; De Rosa, V.; Galgani, M.; Di Rella, F.; Spagnuolo, M. I.; Colamatteo, A.; Fusco, C.; Micillo, T.; Bruzzaniti, S.; Ceriello, A.; Puca, A. A.; Matarese, G. Type 2 Diabetes: How Much of an Autoimmune Disease? Front. Endocrinol. 2019, 10, 348.

18. Gutierrez-Arcelus, M.; Rich, S. S.; Raychaudhuri, S. Autoimmune diseases - connectingrisk alleles with molecular traits ofthe immune system. Nature Reviews Genetics 2016, 17, 160-174.

19. Sakaguchi, N.; Takahashi, T.; Hata, H.; Nomura, T.; Tagami, T.; Yamazaki, S.; Sakihama, T.; Matsutani, T.; Negishi, I.; Nakatsuru, S.; Sakaguchi, S. Altered thymic T-cell selection due to a mutation of the ZAP-70 gene causes autoimmune arthritis in mice. Nature Cell Biology 2003, 426, 454-460.

20. Anderton, S. M. Avoiding autoimmune disease - T cells know their limits. Trends in Immunology 2006, 27, 208-214.

21. Chan, A. C.; Behrens, T. W. Personalizing medicine for autoimmune and inflammatory diseases. Nature Immunology 2013, 14, 106-109.

22. Odegaard, J. I.; Chawla, A. The Immune Systemas a Sensor of the Metabolic State. Immunity 2013, 38, 644654.

23. Pearce, E. L.; Pearce, E. J. Metabolic Pathwaysin Immune Cell Activation and Quiescence. Immunity 2013, 38, $633-643$.

24. Rivera, J. F.; Costes, S.; Gurlo, T.; Glabe, C. G.; Butler, P. C. Autophagy defends pancreatic $\beta$ cells from human islet amyloid polypeptide-induced toxicity. J. Clin. Invest. 2014, 124, 3489-3500.

25. Samuel, S. M.; Varghese, E.; Varghese, S.; Büsselberg, D. Cancer Treatment Reviews. Cancer Treatment Reviews 2018, 70, 98-111.

26. Back, S. H.; Kaufman, R. J. Endoplasmic Reticulum Stress and Type 2 Diabetes. Annu. Rev. Biochem. 2012, 81, 767-793.

27. Lutz, T. A.; Meyer, U. Amylin at the interface between metabolic and neurodegenerative disorders. Front. Neurosci. 2015, 9, 793.

28. Mukherjee, A.; Morales-Scheihing, D.; Butler, P. C.; Soto, C. Type 2 diabetes as a protein misfolding disease. Trends in Molecular Medicine 2015, 1-11.

29. Habib, S. L.; Rojna, M. Diabetes and Risk of Cancer. ISRN Oncology 2013, 2013, 1-16.

30. Kanehisa, M.; Goto, S.; Sato, Y.; Furumichi, M.; Tanabe, M. KEGG for integration and interpretation of largescale molecular data sets. Nucleic Acids Research 2011, 40, D109-D114.

31. Mutch, D. M.; Tordjman, J.; Pelloux, V.; Hanczar, B.; Henegar, C.; Poitou, C.; Veyrie, N.; Zucker, J.-D.; 
Clément, K. Needle and surgical biopsy techniques differentially affect adipose tissue gene expression profiles. The American Journal of Clinical Nutrition 2008, 89, 51-57.

32. X, Y.; R, P.; S, T.; C, B.; P, P. Microarray profiling of skeletal muscle tissues from equally obese, non-diabetic insulin-sensitive and insulin-resistant Pima Indians. Diabetologia 2002, 45, 1584-1593.

33. Davis, S.; Meltzer, P. S. GEOquery: a bridge between the Gene Expression Omnibus (GEO) and BioConductor. Bioinformatics 2007, 23, 1846-1847.

34. Smyth, G. K. Linear Models and Empirical Bayes Methods for Assessing Differential Expression in Microarray Experiments. Statistical Applications in Genetics and Molecular Biology 2004, 3, 1-25.

35. Subramanian, A.; Tamayo, P.; Mootha, V. K.; Mukherjee, S.; Ebert, B. L.; Gillette, M. A.; Paulovich, A.; Pomeroy, S. L.; Golub, T. R.; Lander, E. S.; Mesirov, J. P. Gene set enrichment analysis: A knowledge-based approach for interpreting genome-wide expression profiles. Proc Natl Acad Sci USA 2005, 102, 15545.

36. Mi, H.; Poudel, S.; Muruganujan, A.; Casagrande, J. T.; Thomas, P. D. PANTHER version 10: expanded protein families and functions, and analysis tools. Nucleic Acids Research 2016, 44, D336-42.

37. Alexeyenko, A.; Sonnhammer, E. L. L. Global networks of functional coupling in eukaryotes from comprehensive data integration. Genome Research 2009, 19, 1107-1116.

38. Okawa, S.; Angarica, V. E.; Lemischka, I.; Moore, K.; del Sol, A. A differential network analysis approach for lineagespeci. Nature Publishing Group 2015, 1-8.

39. Quackenbush, J. Microarray data normalization and transformation. Nature Genetics 2002, 32, 496-501.

40. Simon, R. Microarray-based expression profiling and informatics. Current Opinion in Biotechnology 2008, 19, 26-29.

41. Ideker, T.; Thorsson, V.; Siegel, A. F.; Hood, L. E. Testing for differentially-expressed genes by maximumlikelihood analysis of microarray data. Journal of Computational Biology 2000, 7, 805-817.

42. Reimers, M. Making Informed Choices about Microarray Data Analysis. PLoS Comput Biol 2010, 6, e1000786.

43. Chen, K.-H.; Wang, K.-J.; Tsai, M.-L.; Wang, K.-M.; Adrian, A. M.; Cheng, W.-C.; Yang, T.-S.; Teng, N.-C.; Tan, K.-P.; Chang, K.-S. Gene selection for cancer identification: a decision tree model empowered by particle swarm optimization algorithm. BMC Bioinformatics 2014, 15, 1-10.

44. Bild, A. H.; Parker, J. S.; Gustafson, A. M.; Acharya, C. R.; Hoadley, K. A.; Anders, C.; Marcom, P. K.; Carey, L. A.; Potti, A.; Nevins, J. R.; Perou, C. M. An integration of complementary strategies for gene-expression analysis to reveal novel therapeutic opportunities for breast cancer. Breast Cancer Res 2009, 11, R55.

45. Salomonis, N.; Hanspers, K.; Zambon, A. C.; Vranizan, K.; Lawlor, S. C.; Dahlquist, K. D.; Doniger, S. W.; Stuart, J.; Conklin, B. R.; Pico, A. R. GenMAPP 2: new features and resources for pathway analysis. BMC Bioinformatics 2007, 8, 217.

46. Girke, T. Microarray Analysis. 2011, 1-42.

47. Lapointe, J.; Li, C.; Higgins, J. P.; van de Rijn, M.; Bair, E.; Montgomery, K.; Ferrari, M.; Egevad, L.; Rayford, W.; Bergerheim, U.; Ekman, P.; DeMarzo, A. M.; Tibshirani, R.; Botstein, D.; Brown, P. O.; Brooks, J. D.; Pollack, J. R. Gene expression profiling identifies clinically relevant subtypes of prostate cancer. Proc. Natl. Acad. Sci. U.S.A. 2004, 101, 811-816.

48. Moradi, A.; Pourseif, M. M.; Jafari, B.; Parvizpour, S.; Omidi, Y. Pharmacological Research. Pharmacological Research 2020, 156, 104790.

49. Asgharzadeh, M. R.; Pourseif, M. M.; Barar, J.; Eskandani, M.; Niya, M. J.; Mashayekhi, M. R.; Omidi, Y. Functional expression and impact of testis-specific gene antigen 10 in breast cancer: a combined in vitro and in silico analysis. Tabriz University of Medical Sciences 2019, 9, 145-159.

50. Saadat, Y. R.; Pourseif, M. M.; Vahed, S. Z.; Barzegari, A.; Omidi, Y.; Barar, J. Modulatory Role of VaginalIsolated Lactococcus lactison the Expression of miR-21, miR-200b, and TLR-4 in CAOV-4 Cells and In Silico 
Revalidation. 2019, 1-14.

51. Kamal, M. A.; Warsi, M. K.; Alnajeebi, A.; Ali, H. A.; Helmi, N.; Izhari, M. A.; Mustafa, S.; Mobashir, M. Gene expression profiling and clinical relevance unravel the role hypoxia and immune signaling genes and pathways in breast cancer: Role of hypoxia and immune signaling genes in breast cancer. jimsa 2020, 1.

52. Krishnamoorthy, P. K. P.; Kamal, M. A.; Warsi, M. K.; Alnajeebi, A. M.; Ali, H. A.; Helmi, N.; Izhari, M. A.; Mustafa, S.; Firoz, A.; Mobashir, M. Informatics in Medicine Unlocked. Informatics in Medicine Unlocked 2020, 20, 100422. 